\title{
The Research on the Effects of Electrical Aging on Trap Distribution in Polyethylene Doped with Nanoparticies
}

\author{
Lijuan $\mathrm{He}^{1,2}$, Shan Wang ${ }^{1,2,}$ Chao Zhu ${ }^{1,2}$, Haiping Xie ${ }^{1,2}$, \\ Dawei $\mathrm{Li}^{1}$ and Chuntian Chen ${ }^{1 \mathrm{a}}$ \\ ${ }^{1}$ Harbin University of Science and Technology, Harbin, 150080, P.R.China \\ ${ }^{2}$ State Key Laboratory Breeding Base of Dielectrics Engineering, \\ Harbin University of Science and Technology, 150080, China \\ achenchuntian730@sohu.com
}

\begin{abstract}
The photo-stimulated discharge (PSD) technology was used to characterize the trap distribution of polyethylene (PE) and the PE that doped with 5\% $\mathrm{MgO}$ before and after electrical aging. For the PE with the aging time increasing, the internal shallow trap of the material gradually reduced and deep trap gradually increased. But for the PE that doped with $5 \% \mathrm{MgO}$ with the aging time increasing, shallow trap gradually increased and the deep trap gradually reduced. Results show that: during the electrical aging process the PE produces a large amount of free radicals which can introduce deep traps. Adding impurity of $\mathrm{MgO}$ can effectively restrain charge moving that injected from electrode, thus to improve the internal electric field distortion of PE.
\end{abstract}

Keywords: polyethylene; space charge; the charge traps; photo stimulated discharge

\section{Introduction}

In recent years, polymer materials represented by $\mathrm{PE}$ is widely used in power cable and high voltage electrical insulation. But PE used for the high voltage insulation is affected by internal factors with structure defects as well as external factors with impurities. The chemical structure and composition of dielectric are changed by means of degradation, oxidation, cross-linking effect under the influence of high electric field and strong magnetic field for a long time, which is followed by electrical branch and then the material aging accelerated. Electrical aging problem of polymer dielectric can not be ignored. So it is particularly important to improve the aging performance by doping modification [1].

Due to all kinds of structural defects of polymer dielectric materials, local area and doping modification introduced by the crystalline area and amorphous area interface etc will contribute to charge traps, charge was captured by the traps and then space charge formed, the electric field of dielectric materials is significantly distorted due to space charge [2]. At the same time, the nature of the trap is closely linked to the storage and transport of charge. So many features of polymer dielectric materials are closely related to the distribution of space charge [3-4]. Therefore, scientific and accurate characterization of trap energy spectrum distribution in the dielectric material is of great scientific significance and practical value to improve the performance and application level of the dielectric material [5].

Numerous studies show that space charge trap is closely related to insulation aging especially space charge going into and taking off from a trap [6]. A certain guiding role is going on understanding the structure and properties of PE of doping modification, at the same 
time, a certain reference value is provided in understanding electrical aging mechanism of polymer dielectric and synthesis modification of insulation materials [7].

\section{Experiment}

The PE and that doped with $5 \% \mathrm{MgO}$ with the thickness of 25 microns were chosen as the sample, and samples were plated with gold electrodes on both sides by vacuum sputtering. First, the unpolarized samples were tested with PSD method. The samples that are first charged with polarization field strength and the polarization time $40 \mathrm{kv} / \mathrm{mm}$ and $60 \mathrm{~min}$ respectively will then be dealt with short circuit processing on plate electrode, which is followed with the PSD test. Then electric aging experiment was applied on samples with the field intensity of aging $20 \mathrm{kv} / \mathrm{mm}$. Every time aging experiment was completed, polarization and short circuit were applied on the samples the same as processing mentioned above, then PSD test was applied again. Samples were irradiated with the exciting wavelength ranging from $420 \mathrm{~nm}$ to $210 \mathrm{~nm}$, scanning step $2 \mathrm{~nm}$ and scanning time $2 \mathrm{~s}$.

The PSD measurement device consists of excitation light source, test sample room, energymeter, electrometers and data acquisition system, the schematic diagram is shown in Figure 1.

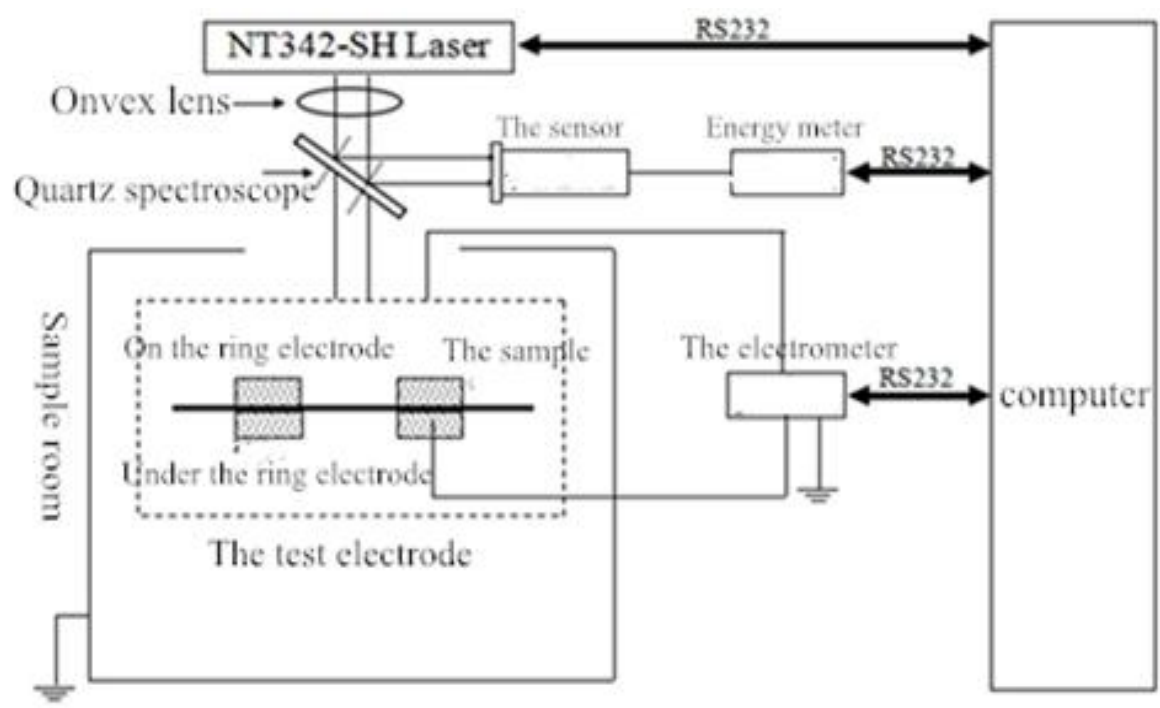

Figure 1. The schematic diagram of PSD

The excitation light source of PSD testing device is wavelength tunable laser (NT342 - SH laser), the automatic adjustment wavelength can be realized by LabVIEW program, after the laser is started, opposite the light outlet and a certain distance from the light outlet is placed a quartz convex lens, the wavelength range needed through the convex lens is split by quartz spectroscope, of which 10 percent of the light reflected to the energy probe through the spectroscope is received by energy sensor and is passed to the energy meter, and then is communicated with the computer at the same time by the preset serial port and data is recorded, while the remaining 90 percent of emergent light through the spectroscope is irradiated onto the sample, the light amplified is irradiated into the ring electrode and the spot diameter is less than or equal to the ring electrode diameter, which is to make full use of the energy of the incident light, to ensure that the total effective photon number is unchanged, and to establish necessary theoretical basis for normalized energy when processing data. 


\subsection{The PSD curve of unpolarized $\mathrm{PE}$ and that doped with $5 \% \mathrm{MgO}$}

The unpolarized sample was placed on the testing electrode and attenuation current was observed through the electrometer, when the current was decayed below to 1 PA the PSD test was started. PSD test was applied to the samples with no charge injected. The real-time current data was collected through the electrometer and finally the PSD curve of PE uncharged and that charged with 60min were curved as shown in Figure 2.

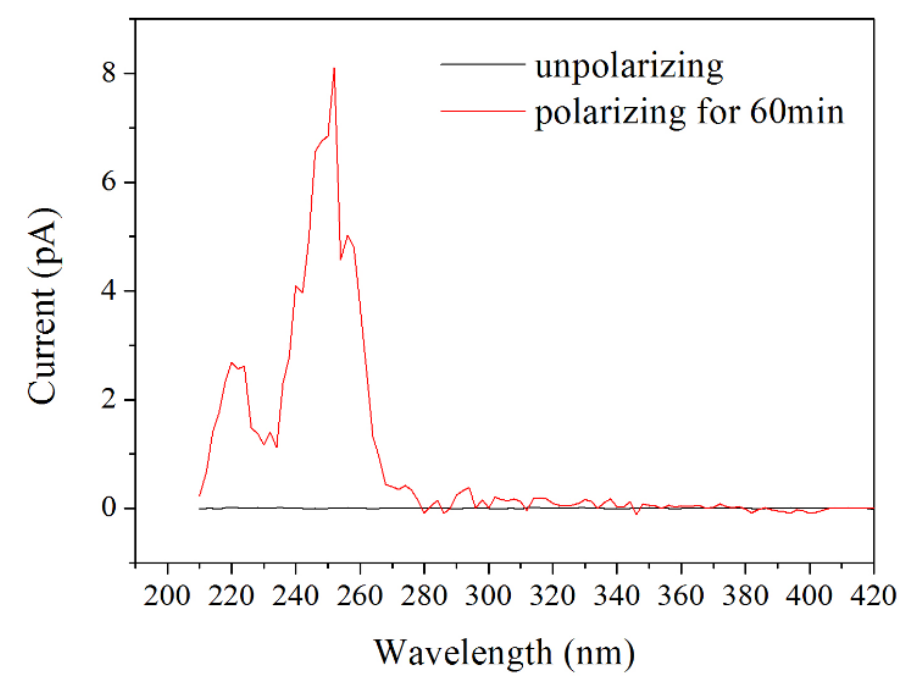

Figure 2. The PSD curve of unpolarized pure PE

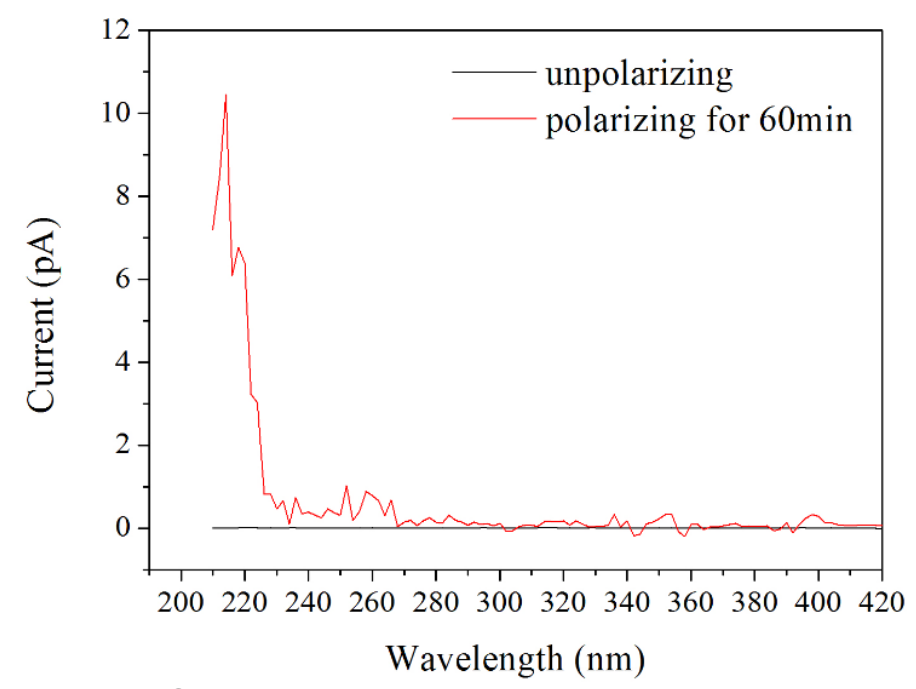

Figure 3. The PSD curve of unpolarized PE doped with $5 \% \mathrm{MgO}$

Then PSD test was applied on unpolarized PE doped with 5\% MgO, specific operating process was the same as the method mentioned above and finally the PSD curve of sample uncharged and that charged with 60min were curved as shown in Figure 3.

\subsection{The PSD test of PE with different aging time}

The electric aging experiment was applied on the PE sample. Every time the aging test was 
completed, the sample was injected with charges for $60 \mathrm{~min}$. As shown in Figure 4 was PSD curve of $\mathrm{PE}$ after aging for $0 \mathrm{~h}, 48 \mathrm{~h}$ and $96 \mathrm{~h}$ respectively.

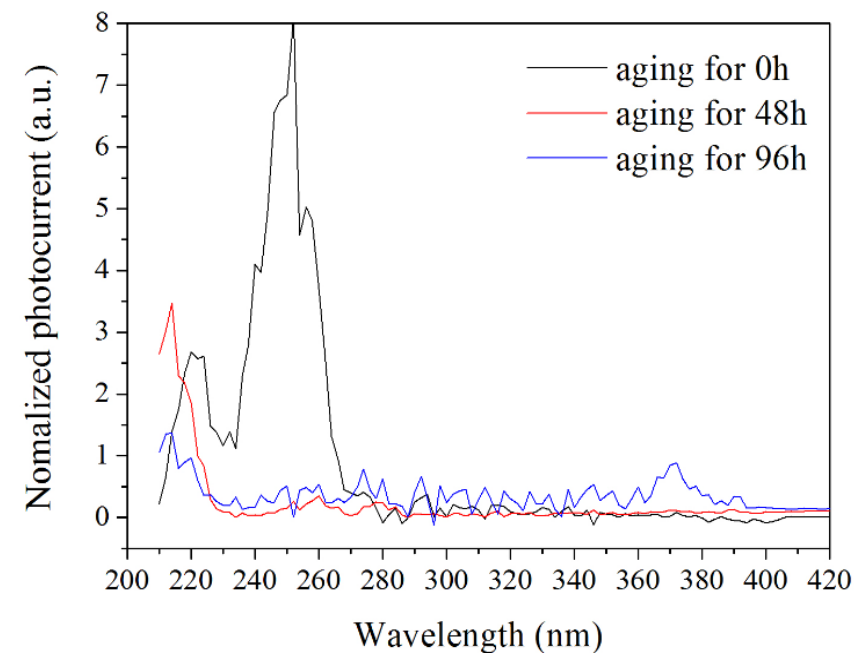

Figure 4. The PSD curve of PE with different aging time

\subsection{The PSD test of PE doped with 5\% MgO with different aging time}

The same as the method used above, the PE doped with $5 \% \mathrm{MgO}$ was tested. As shown in Figure 5 was PSD curve of PE doped with $5 \% \mathrm{MgO}$ after aging for $0 \mathrm{~h}, 24 \mathrm{~h}$ and $36 \mathrm{~h}$ respectively.

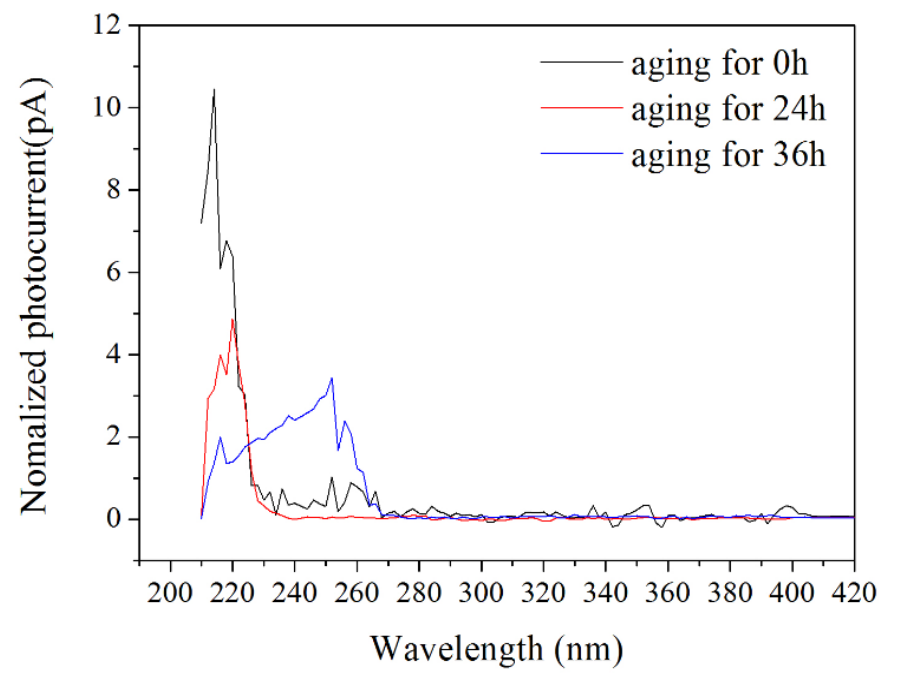

Figure 5. The PSD curve of PE doped with $5 \% \mathrm{MgO}$ with different aging time

\section{Results and Discussion}

As can be seen from Figure 2 and Figure 3, no electric current signal can be collected from peripheral circuit for the two unpolarized samples, which can be used to compare with the PSD spectrum that polarization is applied.

As can be seen from Figure 4, when the unaged PE sample is scanned From 420nm to 
$210 \mathrm{~nm}$, the current is not obtained from $420 \mathrm{~nm}$ to $280 \mathrm{~nm}$ while it is found from $280 \mathrm{~nm}$ to $210 \mathrm{~nm}$. The trap energy level can be regarded as zonal distribution as a whole and the trap depth is calculated to range from $4.4 \mathrm{eV}$ to $5.9 \mathrm{eV}$ and the current reaches the maximum at $252 \mathrm{~nm}$ with the corresponding trap depth $4.9 \mathrm{eV}$;

When the PSD testing is applied on samples after aging for $48 \mathrm{~h}$, the current is not obtained from $420 \mathrm{~nm}$ to $228 \mathrm{~nm}$ while it is found from $228 \mathrm{~nm}$ to $210 \mathrm{~nm}$. The trap depth is calculated to range from $5.4 \mathrm{eV}$ to $5.9 \mathrm{eV}$ and the current reaches the maximum at $218 \mathrm{~nm}$ with the corresponding trap depth $5.7 \mathrm{eV}$;

When the PSD testing is applied on samples after aging for $96 \mathrm{~h}$, the current is not obtained from $420 \mathrm{~nm}$ to $275 \mathrm{~nm}$ while it is found from $275 \mathrm{~nm}$ to $210 \mathrm{~nm}$. The trap depth is calculated to range from $4.5 \mathrm{eV}$ to $5.9 \mathrm{eV}$ and the current reaches the maximum at $214 \mathrm{~nm}$ with the corresponding trap depth $5.8 \mathrm{eV}$.

As can be seen from Fig.5, when the unaged PE sample doped 5\% $\mathrm{MgO}$ is scanned From $420 \mathrm{~nm}$ to $210 \mathrm{~nm}$, the current is not obtained from $420 \mathrm{~nm}$ to $228 \mathrm{~nm}$ while it is found from $228 \mathrm{~nm}$ to $210 \mathrm{~nm}$. the trap depth is calculated to range from $5.4 \mathrm{eV}$ to $5.9 \mathrm{eV}$ and the current reaches the maximum at $214 \mathrm{~nm}$ with the corresponding trap depth $5.8 \mathrm{eV}$;

When the PSD testing is applied on sample after aging for $24 \mathrm{~h}$, the current is not obtained from $420 \mathrm{~nm}$ to $228 \mathrm{~nm}$ while it is found from $228 \mathrm{~nm}$ to $210 \mathrm{~nm}$. Compared with the unaged sample, trap density decreases significantly, the trap depth is calculated to range from $5.4 \mathrm{eV}$ to $5.9 \mathrm{eV}$ and the current reaches the maximum at $220 \mathrm{~nm}$ with the corresponding trap depth $5.6 \mathrm{eV}$;

When the PSD testing is applied on samples after aging for $36 \mathrm{~h}$, the current is not obtained from $420 \mathrm{~nm}$ to $266 \mathrm{~nm}$ while it is found from $266 \mathrm{~nm}$ to $210 \mathrm{~nm}$. The trap depth is calculated to range from $4.7 \mathrm{eV}$ to $5.9 \mathrm{eV}$ and the current reaches the maximum at $252 \mathrm{~nm}$ with the corresponding trap depth $4.9 \mathrm{eV}$.

\section{Summary}

PSD technology was used to test the PSD energy spectrum of the PE and the PE that doped with $5 \% \mathrm{MgO}$ before and after electrical aging. It can be seen through the above analysis that: for the PE with the aging time increasing, the internal shallow trap of the material gradually reduced and deep trap gradually increased. But for the PE that doped with 5\% $\mathrm{MgO}$, with the aging time increasing, shallow trap gradually increased and the deep trap gradually reduced. Results show that: during the electrical aging process the pure PE produces a large amount of free radicals which can introduce deep traps. Adding impurity of $\mathrm{MgO}$ can effectively restrain charge moving that injected from electrode, thus to improve the internal electric field distortion of PE.

\section{Acknowledgments}

The authors would like to express sincere gratitude to all the colleagues in our project group. This work was the program supported by Natural Science Foundation of Heilongjiang under Contract No. E200720, F201301, the program of Educational Commission of Heilongjiang Province under Contract No. 12521079 and Heilongjiang Province graduate entrepreneurship research project in 2012 under Contract No. HLJ YJSCX2012-119.

\section{Reference}

[1] G. Chen, M. Fu, X. Z. Liu and L. S. Zhong, "AC aging and space-charge characteristic in low-density polyethylene polymeric insulation", Journal of applied physics, vol. 97, no. 8, (2005), pp. 837-843.

[2] G. Mazzanti and G. C. Montanari, "Electrical aging and life models the role of space charge", IEEE, 
Transaction on Dielectrics and Electrical Insulation, vol. 12, no. 5, (2005), pp. 876-890.

[3] Y. Maeno, N. Hirai, Y. Ohki, T. Tanaka, M. Okashita and T. Maeno, "Effects of cross-linking byproducts on space charge formation in cross-linking polyethylene", IEEE, Transactions on Dielectrics and Electrical Insulation, vol. 12, no. 1, (2005), pp. 90-97.

[4] J. Castellon, H. N. Nguyen, S. Agnel, A. Toureille and M. Frechette, "Electrical and thermal aging of microcomposite nano-filled epoxy: influence on space charge accumulation", IEEE, Conference on Electrical Insulation and Dielectric Phenomena, Virginia, U.S.A., (2009), October 18-21.

[5] Z. E. Zhu, Y. W. Zhang, Z. L. An and F. H. Zheng, "Trap levels in low density polyethylene doped with nanoparticles by photo-stimulated discharger", Acta Phys. Sin, vol. 61, no. 6, (2012), pp. 543-549.

[6] A. Mellinger and F. C. Gonzales, "Photo-Stimulated Discharge in Electret Polymers an Alternative Approach for Investigating Deep Traps", IEEE, Trans. on DEI., vol. 11, no. 2, (2004), pp. 218-226.

[7] L. He, L. Dai, C. Yang, F. Tian, L. Zhao, J. Cao, X. Wang and Q. Lei, "Study on Deep Trap States in Polymers by Photo-stimulated Discharge Based on the OPO Laser”, Modern Physics Letters B. vol. 24, no. 18, (2010), pp. 1933-1941. 